\title{
Prandtl Number Effects on Mixed Convection Between Rotating Coaxial Disks
}

\author{
CHYI-YEOU SOONG \\ Professor, Department of Aeronautical Engineering, Chung Cheng Institute of Technology, Tahsi, Taoyuan, Taiwan 33509, \\ Republic of China
}

(Received August 9, 1995)

\begin{abstract}
Prandtl number characterizes the competition of viscous and thermal diffusion effects and, therefore, is an influential factor in thermal-fluid flows. In the present study, the Prandtl number effects on non-isothermal flow and heat transfer between two infinite coaxial disks are studied by using a similarity model for rotation-induced mixed convection. To account for the buoyancy effects, density variation in Coriolis and centrifugal force terms are considered by invoking Boussinesq approximation and a linear density-temperature relation. Co-rotating disks $\left(\Omega_{2}=\Omega_{1}\right)$ and rotor-stator system $\left(\Omega_{1} \neq \Omega_{2}=\right.$ $0)$ are considered to investigate the free and mixed convection flows, respectively. For Reynolds number, Re, up to 1000 and the buoyancy parameter, $\mathrm{B} \equiv \beta \Delta \mathrm{T}$, of the range of $|\mathrm{B}| \leq 0.05$, the flow and heat transfer characteristics with Prandtl numbers of $100,7,0.7,0.1$, and 0.01 are examined. The results reveal that the Prandtl number shows significant impact on the fluid flow and heat transfer performance. In the typical cases of mixed convection in a rotor-stator system with $|\mathrm{B}|=$ 0.05 , the effects in buoyancy-opposed flows $(B=0.05)$ are more pronounced than that in buoyancy-assisted ones.
\end{abstract}

Key Words: Prandtl number effects; rotation-induced buoyancy; mixed convection; similarity solutions; two-disk problem

\section{INTRODUCTION}

$\mathrm{T}$ he rotating-disk flow is related to a number of fundamental issues in fluid dynamics as well as to the practice of a variety of rotating machinery. After the pioneering work with the similarity analysis for the free-disk flow by von Karman [1921], and the analyses concerned with flows between coaxial disks by Batchelor [1951] and Stewartson [1953], numerous subsequent investigators aimed at the solutions of the simple models of similarity nature for the two-disk problems, e.g. Lance and Rogers [1962], Pearson [1965], Mellor et al. [1968] and Barrett [1975] etc.

By considering non-uniformity of the fluid temperature in a rapidly rotating device, rotation-induced buoyancy effect may become important for high rotational forces. The rotational buoyancy, centrifugal and/or Coriolis, have been included in some previous studies on rotating systems, e.g. the rotating closed cylinders by Busse and Carrigan [1974], Homsy and Hudson [1969a,b], Hudson et al. [1978], Guo and Zhang [1992], gas centrifuges by Matsuda et al. [1976], Matsuda and
Hashimoto [1976], and radially rotating channels by Siegel [1985], Soong and Hwang [1990, 1993]. In the presence of a through-flow in wheel-space of the finite coaxial disks, the rotation-induced buoyancy effects have been studied by solutions of bounday-layer equations (Soong and Yan [1993]) and Navier-Stokes equations (Soong and Tzong [1991]). For infinite disks without through-flow, Hudson [1968] has performed an analysis for flow between two co-rotating disks with consideration of the rotational buoyancy. Only the low Reynolds numbers $(\operatorname{Re} \leq 100)$ and very small buoyancy parameters $(\beta \Delta \mathrm{T} \leq 0.01)$ were involved in the analysis. Later, Chew [1981] developed a linearized model by neglecting radial viscous terms and nonlinear inertia terms in momentum equations. However, the solution is essentially a highly simplified one. Most recently, by solely considering centrifugal buoyancy effect, a unified similarity analysis for the free, forced, and mixed convection flow and heat transfer in two-disk problems has been developed [1995].

In thermal-fluid flows, Prandtl number characterizes the competition of the viscous and the thermal diffusion 
effects. Therefore, it can be expected that the Prandtl number plays an influential role in the free and mixed convection flow and heat transfer problems. In the present study, a similarity model of rotation-induced buoyancy is employed and the emphasis is placed on the Prandtl number effects on flow and heat transfer characteristics in this class of buoyancy-influenced rotating flows. Two rotational conditions, i.e. the co-rotating disks $\left(\Omega_{2}=\Omega_{1}\right)$ and the rotor-stator system $\left(\Omega_{1} \neq \Omega_{2}=\right.$ $0)$ are considered. For Reynolds number up to 1000 and buoyancy parameter in the range of $-0.05 \leq \beta \Delta \mathrm{T} \leq$ 0.05 , the flow and heat transfer characteristics with $\operatorname{Pr}=$ $100,7,0.7,0.1$, and 0.01 are examined.

\section{THEORETICAL ANALYSIS}

\section{Problem Statement}

Two infinite coaxial disks separated by a spacing $S$ as shown in Figure 1 is the physical model of the problem. Two disks rotating at rotational rates $\Omega_{1}$ and $\Omega_{2}$ are of uniform temperatures $T_{1}$ and $T_{2}$, respectively. A cynlindrical coordinate $(R, \varphi, Z)$ is fixed on the disk 1 and its origin lies at the disk center. With respect to the rotating frame shown in Figure 2, the fluid particle locating at a radial distance $\mathrm{R}$ away from the axis of rotation and rotating at $\Omega \mathbf{k}$ encounters three rotational body forces, i.e. the centrifugal force, $\rho R \Omega^{2}$, radial component, $2 \rho \Omega V$, and circumferential component, $2 \rho R \Omega U$, of the Coriolis force. The fluid flow is assumed to be steady, laminar, axi-symmetric and of constant-property; and the Boussinesq approximation is invoked to take into account the rotation-induced buoyancy effect. The stress-work effects are all ignored. The gravitational force term is also neglected by comparing with the centrifugal force in rapidly rotating systems. In the present study, the wall condition of disk 1 is used as the reference state, at which the fluid confined by the disks lies at the temperature $T_{r}$ $=\mathrm{T}_{1}$ and rotates with the reference frame as a solid body, therefore, $\mathrm{U}=\mathrm{V}=\mathrm{W} \equiv 0$ and $-\nabla \mathrm{P}_{\mathrm{r}} / \rho_{\mathrm{r}}=\boldsymbol{\Omega} \times \boldsymbol{\Omega} \times$ R. Furthermore, by considering a linear density-temperature relation, $\rho=\rho_{\mathrm{r}}\left[1-\beta\left(\mathrm{T}-\mathrm{T}_{\mathrm{r}}\right)\right]$, the governing

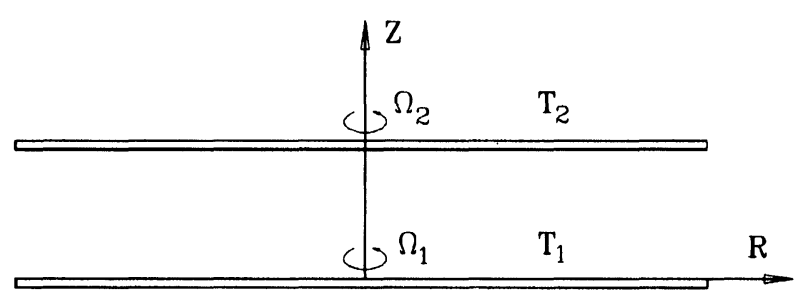

FIGURE 1 Physical model of two co-axially rotating infinite disks.

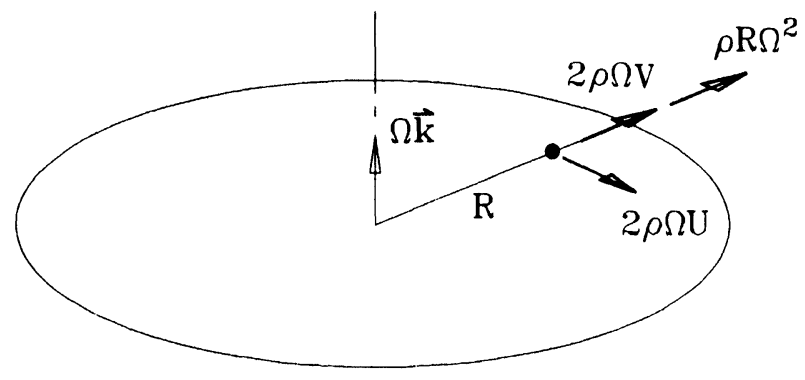

FIGURE 2 Rotational forces acting on the moving fluid particle.

equations can be depicted in a similar form as that in the work of Homsy and Hudson [1969]:

$$
\nabla \cdot \mathrm{V}=0
$$

$$
\begin{aligned}
& (\mathbf{V} \cdot \nabla) \mathbf{V}+2 \Omega_{1}\left[1-\beta\left(\mathrm{T}-\mathrm{T}_{\mathrm{r}}\right)\right](\mathbf{k} \times \mathbf{V})+\Omega_{1}{ }^{2} \mathrm{R} \beta(\mathrm{T} \\
& \left.-\mathrm{T}_{\mathrm{r}}\right) \mathbf{i}=-\nabla \mathrm{P}^{\prime} / \rho_{\mathrm{r}}+\nu \nabla^{2} \mathbf{V}
\end{aligned}
$$

$$
(\mathbf{V} \cdot \nabla) \mathrm{T}=\alpha \nabla^{2} \mathrm{~T}
$$

in which $\mathrm{P}^{\prime}=\mathrm{P}-\mathrm{P}_{\mathrm{r}}$ is the pressure departure from the reference condition, $\Omega=\Omega_{1} \mathbf{k}, \mathbf{R}=\mathrm{R} \mathbf{i}$, and $\mathbf{k}$ and $\mathbf{i}$ are the unit vectors in the axial and radial directions, respectively. By using the following dimensionless variables and parameters

$$
\begin{aligned}
& \mathrm{F}(\eta)=\mathrm{U}(\mathrm{R}, \mathrm{Z}) / \mathrm{R} \Omega_{1}, \mathrm{G}(\eta)=\mathrm{V}(\mathrm{R}, \mathrm{Z}) / \mathrm{R} \Omega_{1}, \\
& \mathrm{H}(\eta)=\mathrm{W}(\mathrm{R}, \mathrm{Z}) /\left(\nu \Omega_{1}\right)^{1} /^{2} \\
& \theta(\eta)=\left[\mathrm{T}(\mathrm{R}, \mathrm{Z})-\mathrm{T}_{1}(\mathrm{R})\right] / \Delta \mathrm{T}, \\
& \eta=\mathrm{Z} / \mathrm{S}, \mathrm{R} e=\Omega_{1} \mathrm{~S}^{2} / \nu, \mathrm{P} r=\nu / \alpha, \mathrm{B}=\beta \Delta \mathrm{T}
\end{aligned}
$$

where $\mathrm{U}, \mathrm{V}$ and $\mathrm{W}$, respectively, represent velocity components in $\mathrm{R}, \varphi$ and $\mathrm{Z}$ directions, and $\Delta \mathrm{T}$ is the characteristic temperature difference. Equations (1-3) can be recast into the following dimensionless form:

$$
\begin{gathered}
\mathrm{H}^{\prime \prime \prime}=\mathrm{R} e^{1 / 2} \mathrm{HH}^{\prime \prime \prime}+4 \mathrm{R} e^{3 / 2}\left[(1+\mathrm{G}) \mathrm{G}^{\prime}-\mathrm{B}\left(\mathrm{G}^{\prime} \theta+\mathrm{G} \theta^{\prime}\right)\right] \\
-2 \mathrm{BR} e^{3 / 2} \theta^{\prime} \\
\mathrm{G}^{\prime \prime}=\mathrm{R} e^{1 / 2}\left[\mathrm{HG}^{\prime}-\mathrm{H}^{\prime}(1+\mathrm{G})+\mathrm{BH}^{\prime} \theta\right] \\
\theta^{\prime \prime}=\operatorname{Pr} \operatorname{Re}^{1 / 2} \mathrm{H} \theta^{\prime} .
\end{gathered}
$$

The relationship between axial and radial velocities:

$$
\mathrm{H}^{\prime}+\mathrm{R} e^{1 / 2} \mathrm{~F}=0
$$


has been introduced to the above system. The Z-component of momentum equation, with a dimensionless pressure parameter $\Pi \equiv \mathrm{P}^{\prime} / \rho_{\mathrm{r}} \mathrm{S}^{2} \Omega_{1}{ }^{2}$, can be written as

$$
\Pi=\mathrm{R} e^{-3 / 2} \mathrm{H}^{\prime}-\mathrm{R} e^{-1} \mathrm{H}^{2} / 2
$$

Obviously, Equation (9) is not coupled with the system of Equations (5-7). After solving the axial velocity solution $\mathrm{H}(\eta)$, the pressure function $\Pi$ can be evaluated. The boundary conditions for the velocity and temperature functions are

$$
\begin{aligned}
& \mathrm{H}(0)=\mathrm{H}^{\prime}(0)=\mathrm{H}(1)=\mathrm{H}^{\prime}(1)=0 \\
& \mathrm{G}(0)=\mathrm{G}(1)-\gamma=0 \\
& \theta(0)=\theta(1)-1=0
\end{aligned}
$$

where the parameter $\gamma \equiv\left(\Omega_{2}-\Omega_{1}\right) / \Omega_{1}$ denotes the dimensionless rotation rate of the disk 2 . Note that the boundary conditions for $F(\eta), F(0)=F(1)=0$, have been already replaced by $H^{\prime}(0)=H^{\prime}(1)=0$ through Equation (8).

\section{Governing Parameters}

Four parameters are involved in the problem, they are Pr, $\mathrm{Re}, \mathrm{B}$, and $\gamma$. The Prandtl number indicates the relative importance of viscous to thermal diffusion effects. The Reynolds number $\mathrm{Re}$ characterizes the rotational effect and the thermal Rossby number B measures the buoyancy effect. The last parameter $\gamma$ denotes the relative rotation rate of the disk 2 with respect to that of the disk 1. For example, the values of $\gamma=0$ and -1 correspond to the cases of co-rotating disks $\left(\Omega_{2}=\Omega_{1}\right)$ and rotorstator $\left(\Omega_{1} \neq \Omega_{2} \equiv 0\right)$, respectively. Note that, in this two-disk flow configuration, the cases of $\gamma=0$ and $\mathrm{B} \neq$ 0 are the pure free-convection. While the forced convection is characterized by $\gamma \neq 0$ and $\mathrm{B}=0$. For the non-zero $\mathrm{B}$ as well as $\gamma$, the problem becomes a mixed convection one, in which Re can be used to characterize the forced flow effect. In the conventional free-convection study, for the validity of Boussinesq approximation, $\beta \Delta \mathrm{T}$ was usually small, for example, the magnitude less than 0.1 in the study of Gray and Giorgini [1976]. The positive value of $B$ implies $T_{1}<T_{2}$ and the temperature of the fluid adjacent to the disk 1 is higher than $T_{1}$, the flow is designated as buoyancy-opposed flow: On the contrary, the flow with $\mathrm{B}<0$ (or $\mathrm{T}_{1}>\mathrm{T}_{2}$ ) is a buoyancy-assisted one. In the present study, the parameter $\mathrm{B}$ is restricted in the range of $|\mathrm{B}| \leq 0.05$, the Reynolds number based on the disk spacing lies up to 1000 , and the rotation parameter $\gamma=0$ and -1 are considered.

\section{Friction Factors and Nusselt Numbers}

Based on the definition $\mathrm{C}_{\mathrm{f}} \equiv \tau_{\mathrm{w}} /\left[\rho\left(\mathrm{R} \Omega_{1}\right)^{2} / 2\right]$, where $\rho_{\mathrm{w}}$ denotes wall shear stress, the radial and tangential friction factors at disks 1 and 2 are

$$
\begin{aligned}
& \mathrm{C}_{f r 1} \mathrm{R} e^{*}=2 \mathrm{~F}^{\prime}(0), \mathrm{C}_{f r 2} \mathrm{R} e^{*}=-2 \mathrm{~F}^{\prime}(1), \\
& \mathrm{C}_{f t 1} \mathrm{R} e^{*}=2 \mathrm{G}^{\prime}(0), \mathrm{C}_{f t 2} \mathrm{R} e^{*}=-2 \mathrm{G}^{\prime}(1)
\end{aligned}
$$

respectively. Where $\mathrm{Re}^{*} \equiv\left(\mathrm{R} \Omega_{1}\right) \mathrm{S} / v$ is the local Reynolds number.

Heat transfer performance is characterized by Nusselt number defined as $\mathrm{Nu} \equiv-(\partial \mathrm{T} / \partial \mathrm{n})_{\mathrm{w}} /\left(\mathrm{T}_{2}-\mathrm{T}_{1}\right)$. By this definition the positive and negative values of $\mathrm{Nu}$ denote the heat transferred from and to the wall, respectively.The heat transfer rates on the two disks are $\mathrm{Nu}_{1}$ and $\mathrm{Nu}_{2}$, viz.

$$
\mathrm{N} u_{1}=-\theta^{\prime}(0), \mathrm{N} u_{2}=\theta^{\prime}(1)
$$

\section{NUMERICAL PROCEDURE}

The system of Equations (5-7) with boundary conditions (10) consist of a nonlinear eighth-order two-point boundary value problem. A typical shooting method can be started with the guessed missing conditions: $\mathrm{H}^{\prime \prime}(0)=\mathrm{a}$, $\mathrm{H}^{\prime \prime \prime}(0)=\mathrm{b}, \mathrm{G}^{\prime}(0)=\mathrm{c}$ and $\theta^{\prime}(0)=\mathrm{d}$. In an iterative procedure, the values of $a, b, c$ and $d$ are updated continuously using Newton's method until the boundary conditions at $\eta=1$, i.e. $H(1)=H^{\prime}(1)=G(1)-\gamma=\theta(1)$ $-1=0$, are met. The iteration is regarded as convergent if the stopping criterion, $\max (\Delta \mathrm{a}, \Delta \mathrm{b}, \Delta \mathrm{c}, \Delta \mathrm{d}) \leq 10^{-8}$, is satisfied. Low-Re solutions can be easily obtained using conventional shooting techniques. However, due to the stiffness of the system, the convergent solution is getting hard as Reynolds number increases. By applying nonuniform grid, under-relaxation, and the Aitkin acceleration technique, the convergent solutions at high Reynolds numbers can be attained. Through the numerical experiments, the grid-dependence of the numerical solutions were examined. In general, the grid of 201 points is sufficient for grid-independent solutions. For higher Re, small $|\gamma|$, and/or large $|\mathrm{B}|$, the finer grids, e.g. 400 points or more, were used for either high resolution and convergence of the solutions.

\section{RESULTS AND DISCUSSION}

\section{Flow and Temperature Fields}

In a former study (Soong [1995]), the buoyancy-free solutions of the present formulation have demonstrated 
their reasonable agreement with the experimental results. In the present work the emphasis is placed on the rotation-induced buoyancy effects. Figure 3 shows the free-convection solutions of $\operatorname{Re}=300, \gamma=0$ and $\mathrm{B}=$ 0.05 . For higher $\operatorname{Pr}$, e.g. $\operatorname{Pr}=7$, the temperature distribution deviates from the conductive solutions due to the convection effect. For the increasing temperature gradient near the disk 1 , the cooler fluid is accelerated radially outward. This enhances the peak value of radial velocity which, in turn, alters the axial velocity distribution $\mathrm{H}$. Through the action of the buoyancy effect by Coriolis force the circumferential velocity $G$ presents a noticeable change.

A forced convection (buoyancy-free) solutions for a rotor-stator system $(\gamma=-1)$ with $\operatorname{Re}=500$ and variable Prandtl numbers are presented in Figure 4. Since the velocity-temperature coupling has been broken by ignoring the buoyancy effect, the velocity profiles become independent of the Prandtl number. While the temperature solutions are still a strong function of Pr. For relatively higher Prandtl numbers, e.g. $\mathrm{Pr}=0.7$ and 7 in Figure 4, thermal boundary layer emerges on the disk 1. The appearance of the thermal boundary layer is attributed to the relatively strong convection effect.

Figures 5 and 6 show the buoyancy-influenced counterparts of the case in Figure 4. In Figure 5 with B = 0.05 , the Prandtl number effect significantly alters the flow fields. For large Prandtl number, $\operatorname{Pr}=7$, the temperature function changes abruptly in the thin thermal boundary layer but remain uniform in large portion of the wheel space. As Pr decreases from 7 to 0.01 , the
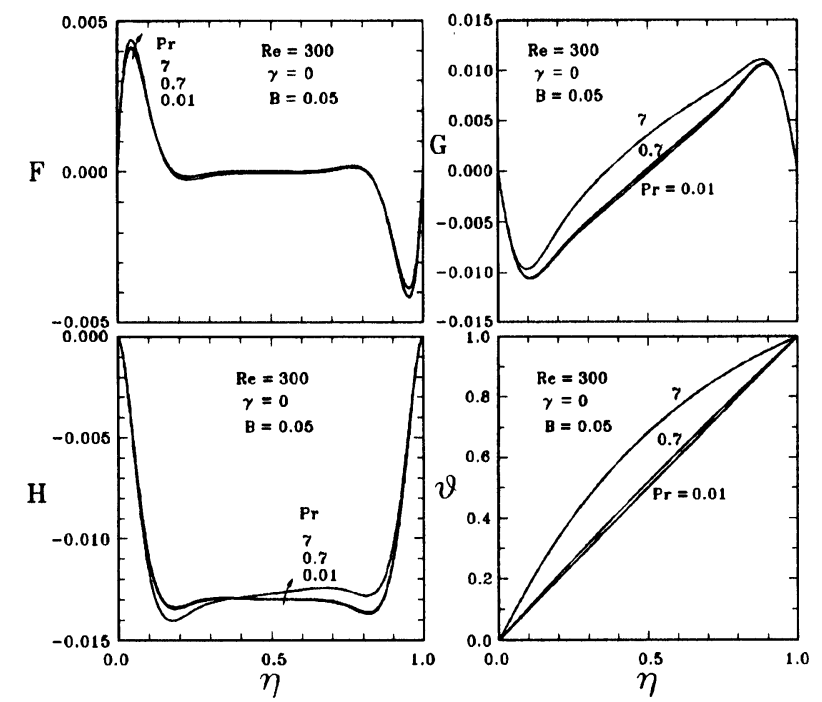

FIGURE 3 Pr-effects on solutions of free convection between corotating disks.
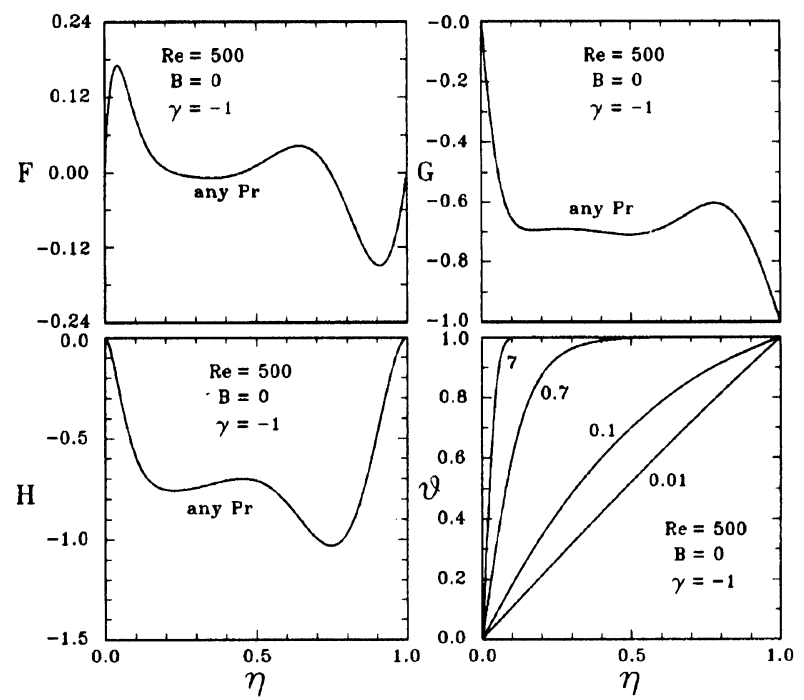

FIGURE 4 Pr-effects on solutions of forced convection in rotor-stator system.

thermal diffusion is getting more and more important and, then, the temperature variation appears notably in the whole domain rather than confined in a narrow region of thermal boundary layer. For small Pr, the temperature gradient near the disk 1 , i.e. $\mathrm{Z}=0$, is alleviated. Therefore, the reduction in buoyancy-opposing effect enhances the radial velocity peak near the disk 1 . The axial velocity distribution is modified with the variation of radial velocity. Due to coupling of the Coriolisinduced buoyancy in circumferential fluid motion and
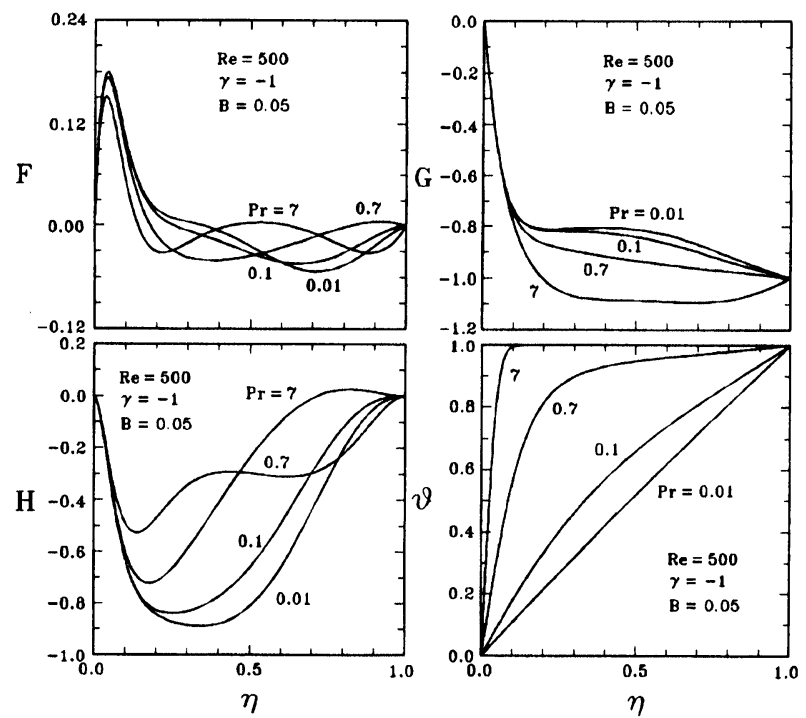

FIGURE 5 Pr-effects on buoyancy-opposed mixed convection in rotor-stator system. 
the Prandtl number effects, salient Pr-dependence of the circumferential velocity is presented. While in the case of buoyancy-assisted flow with $B=-0.05$, the velocity fields are only slightly altered by the change in Prandtl number, see Figure 6, although the temperature distribution varies in the similar manner as that in buoyancy-free and buoyancy-opposed flows.

\section{Friction Factors and Heat Transfer Rates}

Figure 7, as a typical example, shows radial and tangential friction factors in a rotor-stator system with $\mathrm{B}=$ 0.05 . In the region of $R e<100$, friction factors have no significant change with Prandtl number. As the Reynolds number or the rotation-rate increases, the Pr-effect on the friction factors can be enhanced through the remarkable variation in temperature distribution. It is also noteworthy that the changes in radial friction factor is larger than that in tangential one.

Heat transfer performance is measured by the Nusselt number. Figure 8 presents Nusselt numbers on the disks 1 and 2, i.e. $\mathrm{Nu}_{1}$ and $\mathrm{Nu}_{2}$, for buoyancy-assisted flows in a system of $\gamma=-1$ and $B=-0.05$. As that mentioned in the last section, the Prandtl number significantly affects the temperature fields, especially, for moderate/ high Prandtl numbers. In the limit of very small Pr, the heat transfer solution approaches to the conductive state, i.e. $\mathrm{Nu}_{1}=\mathrm{Nu}_{2}=1$. In the rotor-stator system, since the driven force of the mixed convection flow is the rotation
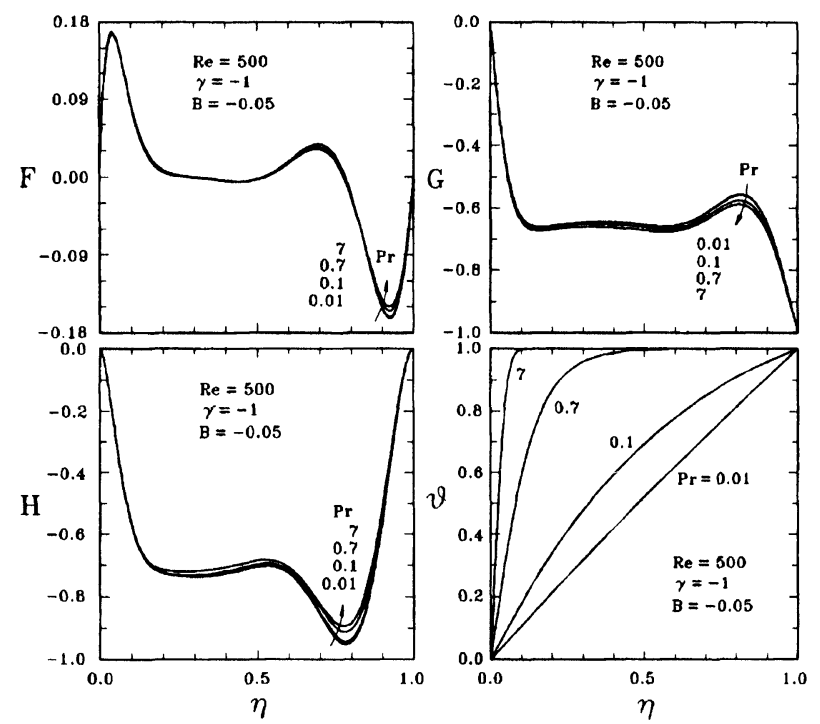

FIGURE 6 Pr-effects on buoyancy-assisted mixed convection in rotor-stator system.

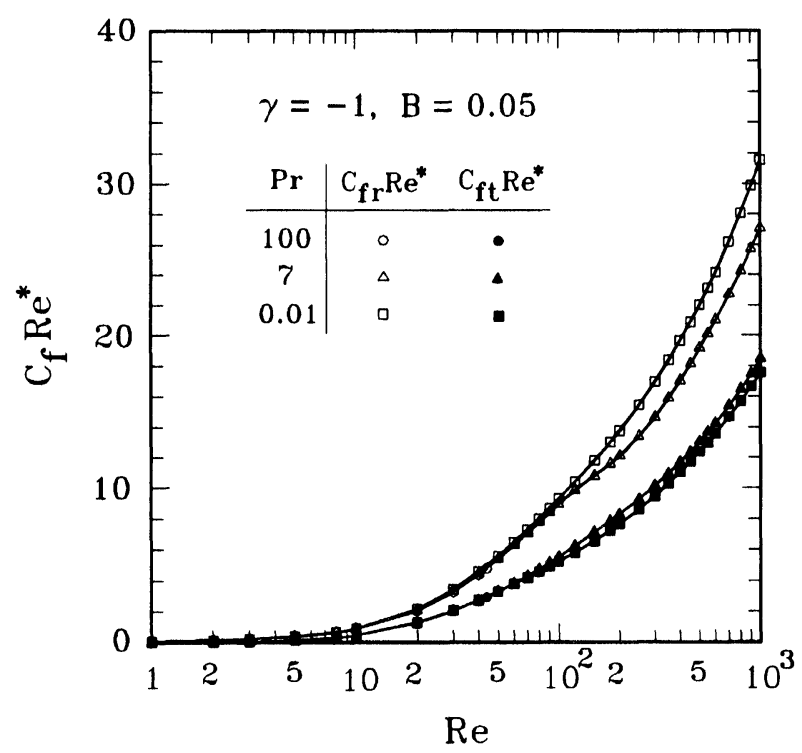

FIGURE 7 Friction factors in mixed convection flows in rotor-stator system.

of the disk 1, the temperature gradient as well as the heat transfer rate are more pronounced on the disk 1 . This is the reason why the value of $\mathrm{Nu}_{1}$ changes drastically but the $\mathrm{Nu}_{2}$ curves appear flat even for a wide range of $\mathrm{Pr}$, i.e. $0.01 \leq \operatorname{Pr} \leq 100$.

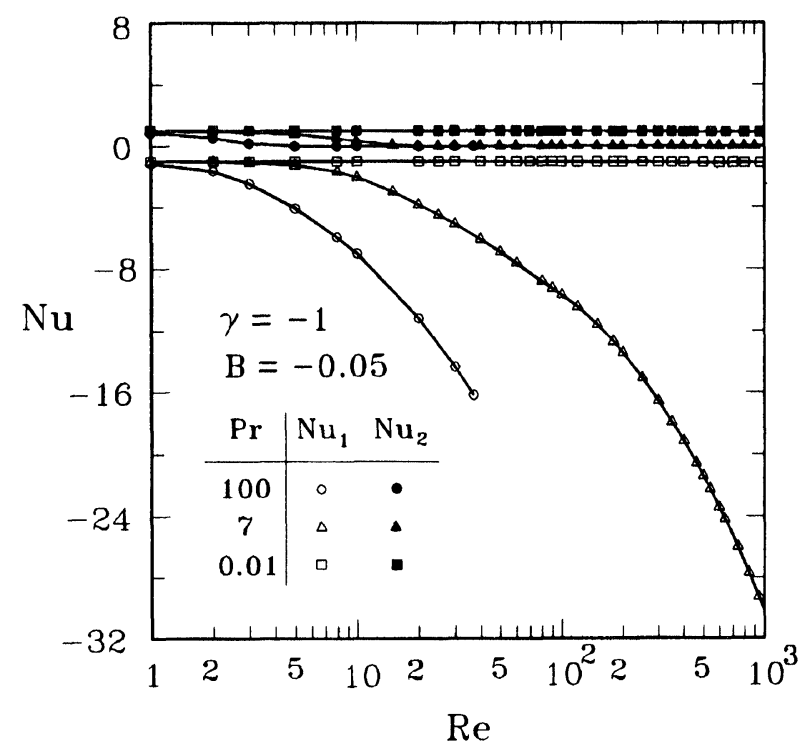

FIGURE 8 Nusselt numbers on disks 1 and 2 for mixed convection in rotor-stator system. 


\section{CONCLUDING REMARKS}

The Prandtl number effects on buoyancy-influenced non-isothermal flow between coaxial disks have been studied by using a similarity model. The results reveal that the change in Pr leads to a variation of temperature distribution, which, in turn, modifies the velocity fields through a coupling of the thermal and hydrodynamic natures linked by the rotation-induced buoyancy effects. From the present theoretical analysis, it can be disclosed that, for the two-disk problems, the Prandtl number presents significant impact on the flow and heat transfer characteristics in either free-convection and mixed convection. In the typical cases of mixed convection flow and heat transfer with the same measure of buoyancy, $|\mathrm{B}|$ $=0.05$, the Pr-effect in buoyancy-opposed flows $(\mathrm{B}=$ $0.05)$ is more pronounced than that in buoyancy-assisted ones $(\mathrm{B}=-0.05)$.

\section{Acknowledgement}

Part of financial support of this study provided by National Science Council, the Republic of China through the grant No. NSC-81-0401-E-211-502 is gratefully acknowledged.

\section{References}

Barrett, K. E., Numerical study of the flow between rotating coaxial discs, Z. Angew. Math. Mech., vol. 26, pp. 807-816, 1975.

Batchelor, G. K., Note on a class of solutions of the Navier-Stokes equations representing steady rotationally-symmetric flow, Quart. J. Mech. Appl. Math., vol. IV, pt. 1, pp. 29-41, 1951.

Busse, F. H., and Carrigan, C. R., Convection induced by centrifugal buoyancy, J. Fluid Mech., vol. 62, pt. 3, pp. 579-592, 1974.

Chew, J. W., Similarity Solutions for Non-Isothermal Flow Between Infinite Rotating Disks, Rept. TFMRC/38, Thermo-Fluid Mech. Res.

Gray, D. D., and Giorgini, A., The Validity of the Boussinesq Approximation for Liquid and Gases, Int. J. Heat Mass Transfer, vol. 19, pp. 545-551, 1976.

Guo, J. Y., and Zhang, C. M., Thermal Drive in Centrifugally Driven Thermal Convection in a Vertical Rotating Cylinder, Int. J. Heat Mass Transfer, vol. 35, pp. 1635-1644, 1992.
Homsy, G. M., and Hudson, J. L., Centrifugally Driven Thermal Convection in a Rotating Cylinder, J. Fluid Mech., vol. 35, pt. 1, pp. 33-52, 1969a.

Homsy, G. M., and Hudson, J. L., Heat Transfer in a Rotating Cylinder of Fluid Heated from Above, Int. J. Heat Mass Transfer, vol. 14, pp. 1149-1159, 1969b.

Hudson, J. L., Non-Isothermal Flow Between Rotating Disks, Chem. Eng. Sci., vol. 23, pp. 1007-1020, 1968b. Centre, School Eng. Appl. Sci, Univ. Sussex, 1981.

Hudson, J. L., Tang, D., and Abell, S., Experiments on Centrifugally Driven Thermal Convection in a Rotating Cylinder, J. Fluid Mech., vol. 86, pt. 1, pp. 147-159, 1978.

Lance, G. N., and Rogers, M. H., The Axially Symmetric Flow of a Viscous Fluid Between Two Infinite Rotating Disk, Proc. Roy. Sci., vol. A 266, pp. 109-121, 1962.

Matsuda, T., and Hashimoto, K., Thermally, Mechanically or Externally Driven Flows in a Gas Centrifuge With an Insulated Horizontal End Plates, J. Fluid Mech., vol. 78, pt. 2, pp. 337-354, 1976.

Matsuda, T., Hashimoto, K., and Takeda, H., Thermally Driven Flow in a Gas Centrifuge With an Insulated Side Wall, J. Fluid Mech., vol. 73, pt. 2, pp. 389-399, 1976.

Mellor, G. L., Chapple, P. J., and Stokes, V. K., On the Flow Between a Rotating and a Stationary Disk, J. Fluid Mech., vol. 31, pt. 1, pp. 95-112, 1968.

Pearson, C. E., Numerical Solutions for the Time-Dependent Viscous Flow Between Two Rotating Coaxial Disks, J Fluid Mech., vol. 21, pt. 4, pp. 623-633, 1965.

Siegel, R., Analysis of Buoyancy Effect on Fully Developed Laminar Heat Transfer in a Rotating Tube, J. Heat Transfer, vol. 107, pp. 338-244, 1985.

Soong, C. Y., Axisymmetric Mixed Convection Between Rotating Coaxial disks, accepted by Int. J. Heat Mass Transfer, 1995

Soong, C. Y., and Hwang, G. J., Laminar Mixed Convection in a Radially Rotating Semiporous Channel, Int. J. Heat Mass Transfer, vol. 33, pp. 1805-1816, 1990.

Soong, C. Y., and Hwang, G. J., Stress Work Effects on Similarity Solutions of Mixed Convection in Rotating Channels With WallTranspiration, Int. J. Heat Mass Transfer, vol. 36, pp. 845-856, 1993.

Soong, C. Y., and Tzong, R. Y., Numerical Investigation of Flow and Heat Transfer in Shrouded Rotor-Stator Systems, Proc. 33rd Aero. Astro. Conf., Taipei, Taiwan, ROC, pp. 831-841, 1991

Soong, C. Y., and Yan, W. M., Numerical Study of Mixed Convection Between Two Symmetrically-Heated Co-Rotating Disks, AIAA J. Thermophysics Heat Transfer, vol. 7, no. 1, pp. 165-170, 1993.

Stewartson, K., On the flow between two rotating coaxial disks, Proc. Camb. Phil. Soc., vol. 3, pp. 333-341, 1953.

von Karman, T., Laminar und turbulente reibung, Z. angew. Math. Mech., vol. 1, pp. 233-244, 1921. 

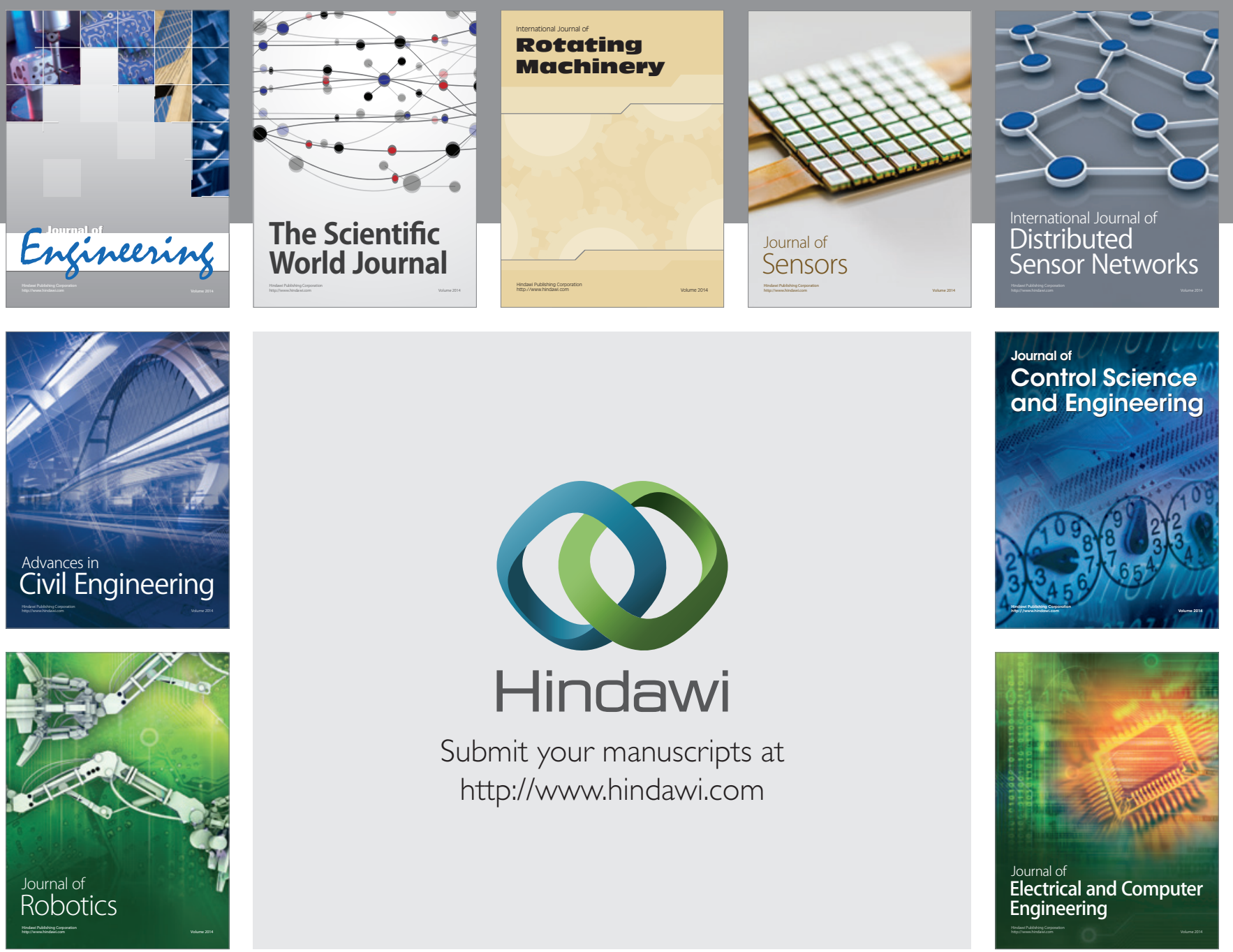

Submit your manuscripts at

http://www.hindawi.com
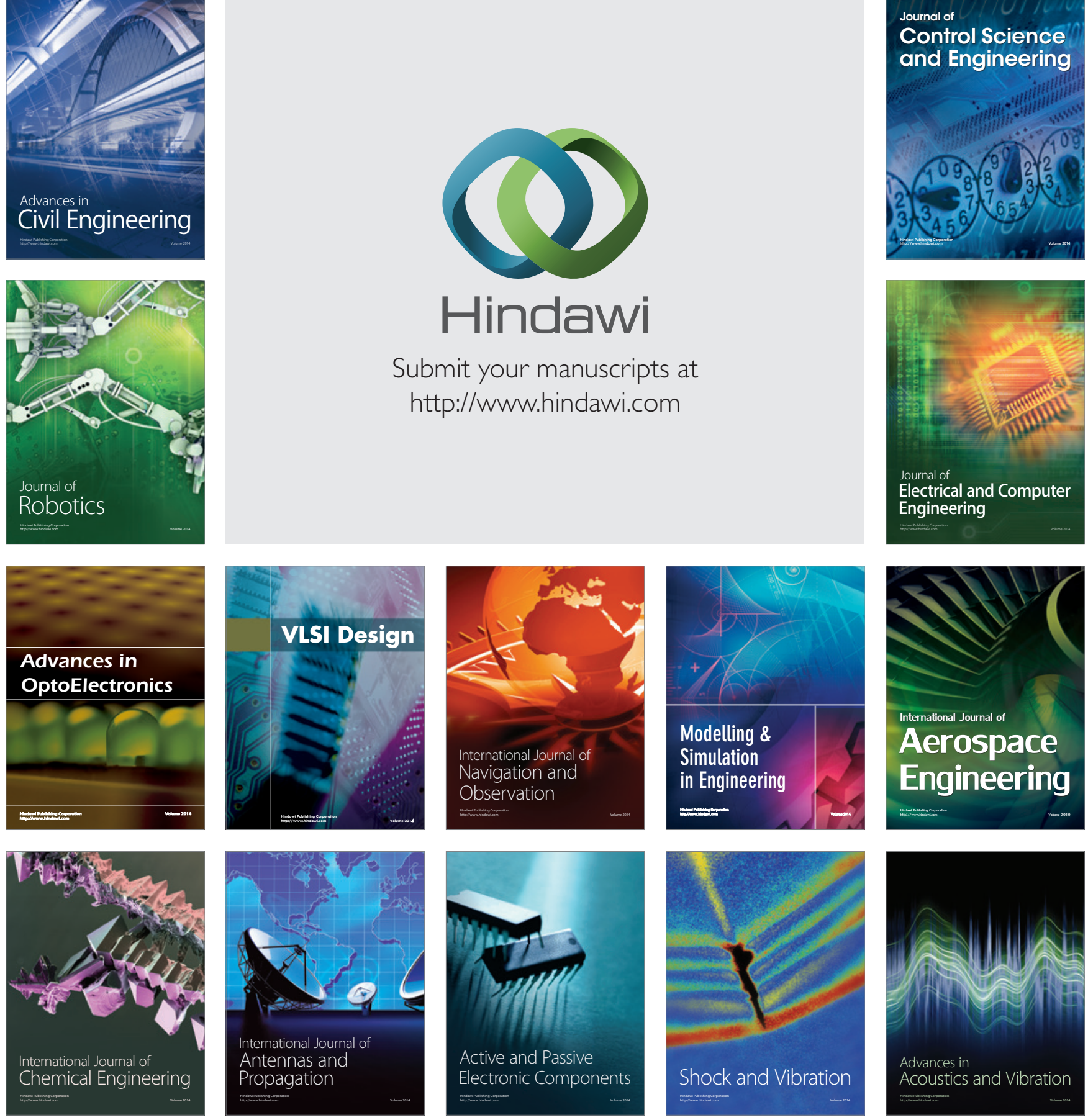